\title{
Exploring ubuntu
}

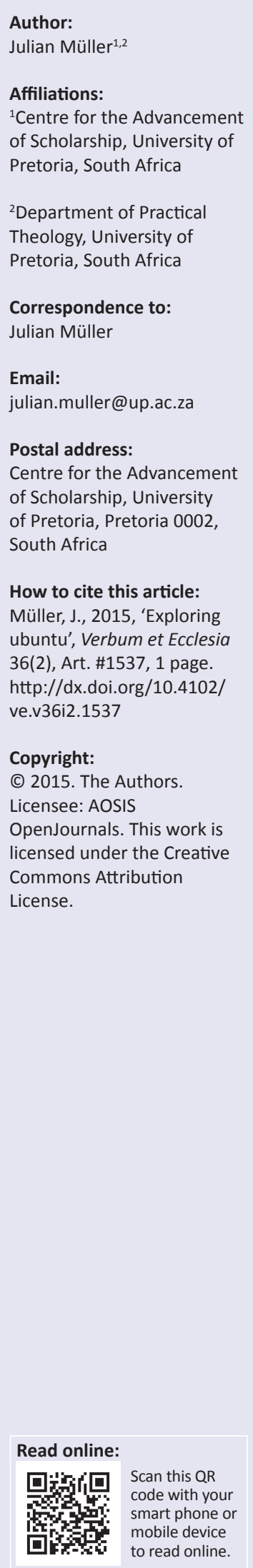

This edition has the aim of opening up space for emerging ideas and theories connected to the Ubuntu Research Project of the University of Pretoria. The project is funded by the Templeton World Aid Foundation, and it runs for three years (2013-2016).

Most of the authors of articles in this special edition are researchers working in Cluster 2 (Theology) of the Project. The others are also connected to the research project. The aim of the articles is not to give a complete overview of the project but to address some of the aspects of this interesting and complicated task. The themes that are explored are all related to the philosophy of ubuntu, but they focus on the background theories and on the thickening of the research story.

Ubuntu, like all philosophies, is not an isolated entity. It is part of a dynamic network of ideas, discourses and cultures. Research on ubuntu can therefore not be done in isolation. It needs to be explored in its relation to other issues like race, gender, ability and disability, and space. The ubuntu story is further thickened with a reflection on nostalgia whilst the root metaphors are also discussed. At least one of the articles grapples with the issue of personal story and bias.

In summary, the aim of this special edition is not to steer the research in any direction or to make claims about an early understanding of ubuntu as personal, spiritual and community value. It is rather an effort to open up the space and thicken the research story as wide as possible in the belief that the opening up of alternative perspectives can only be beneficial to all interested and involved in ubuntu. 